\author{
P Palange \\ SA Ward \\ K-H Carlsen \\ R Casaburi \\ CG Gallagher \\ R Gosselink \\ DE O'Donnell \\ L Puente-Maestu \\ AM Schols \\ S Singh \\ BJ Whipp
}

\section{Recomendações sobre o uso dos testes de exercício na prática clínica}

\section{Recommendations on the use of exercise testing in clinical practice}

\section{Resumo}

A elaboração deste documento pelo grupo de trabalho da European Respiratory Society tem como objectivo apresentar as recomendações sobre o uso clínico dos testes de exercício em doentes com patologia cardiorrespiratória, dando particular ênfase à avaliação funcional, à avaliação do prognóstico e à avaliação das intervenções terapêuticas.

A intolerância ao esforço físico é um dos sintomas mais frequentes, condicionando a perda de qualidade de vida do doente com patologia cardiorrespiratória crónica. Pode definir-se "intolerância ao exercício" numa perspectiva clínica à incapacidade que o doente apresenta para realizar tarefas que os indivíduos saudáveis considerariam toleráveis.

A intolerância ao exercício, considerada em termos do pico de consumo de oxigénio atingido no esforço máximo $\left(V^{\prime} \mathrm{O}_{2 \text { pico }}\right)$ não pode ser prevista por parâmetros avaliados em repouso, como o volume expiratório máximo no primeiro segundo $\left(\mathrm{FEV}_{\mathrm{p}}\right)$, a transferência alvéolo-capilar do monóxido de carbono (DLCO), a fracção de ejecção do ventrículo esquerdo ou o índice de massa corporal (IMC). A avaliação em exercício impõe alguns desafios técnicos: a aplicação de protocolos específicos de incremento de carga de forma precisa e reprodutível, com o recurso habitual a ergómetros, tais como a bicicleta ergométrica e o tapete rolante.

A prova de exercício cardiorrespiratória (CPET) é considerada o gold standard na avaliação das causas de intolerância ao exercício em doentes com doença cardíaca e pulmonar e é baseado no princípio de que a falência do sistema ocorre tipicamente quando o sistema (seja ele músculo-energético, cardiovascular ou pulmonar) se encontra sob stress. A CPET compreende a imposição de um exercício com cargas crescentes (ou seja, incremental) limitado por sintomas, enquanto se monitorizam as variáveis cardiopulmonares (exemplo: consumo de oxigénio $\left(V^{\prime} \mathrm{O}_{2}\right)$, produção de dióxido de carbono $\left(V^{\prime} \mathrm{CO}_{2}\right)$, ventilação minuto $\left(V^{\prime} \mathrm{E}\right)$, frequência cardíaca $(f C)$ ), a percepção de sintomas (exemplo: a dispneia e o desconforto nos membros inferiores) e, quando necessárias, as avaliações da dessaturação arterial do oxigénio relacionada com o esforço, da hiperinsuflação dinâmica e da força muscular dos membros. Os sistemas são forçados até ao seu

ERS Task Force

Eur Respir J 2007; 29: 185-209 
limite tolerável, de forma controlada, o que permite detectar respostas que identificam padrões de alteração e que podem ser relacionadas com padrões de referência previamente estudados e publicados pelas sociedades respiratórias europeia e americanas ${ }^{1-3}$.

Neste documento, é descrito o papel da CPET como auxiliar no diagnóstico e na avaliação funcional e prognóstica. A CPET pode:

- Fornecer uma medição objectiva da capacidade para o exercício;

- Identificar os mecanismos que limitam a tolerância ao exercício;

- Estabelecer índices de prognóstico;

- Monitorizar a progressão da doença e a resposta às intervenções terapêuticas.

- Auxiliar no diagnóstico, em situações de broncoconstrição induzida pelo exercício e de dessaturação arterial do oxigénio.

$\mathrm{Na}$ identificação das causas de intolerância ao exercício, a CPET pode detectar:

- Alterações na entrega de oxigénio (desde a sua entrada nas vias aéreas, passando pelo sistema de transporte cardiocirculatório, até à entrega às mitocôndrias das fibras musculares);

- Limitação ventilatória no exercício;

- Alteração do controlo ventilatório;

- Alteração das trocas gasosas pulmonares;

- Percepção excessiva de sintomas (exemplos: dispneia, precordialgia, fadiga muscular periférica);

- Disfunção metabólica muscular;

- Descondicionamento;

- Fraco esforço dispendido.

Com um bom esforço realizado, se o valor do pico do consumo de oxigénio atingido foi normal e o motivo para parar a prova foi dispneia ou fadiga muscular, então pode considerar-se que o indivíduo estudado tem uma normal tolerância ao exercício. Este cenário irá excluir doença pulmonar (DPOC, doença intersticial pulmonar, doença vascular pulmonar) ou cardíaca (insuficiência cardíaca congestiva) significativas como causa de intolerância.

A prova de exercício cardiopulmonar pode auxiliar no diagnóstico diferencial entre limitação no esforço de origem pulmonar ou cardiocirculatória. Pode fornecer um perfil de respostas que caracterizam determinadas doenças; exemplo: na DPOC são frequentes a limitação ventilatória, a hiperinsuflação dinâmica, a dessaturação arterial com o exercício, a dispneia, a disfunção dos músculos periféricos; na doença intersticial pulmonar são frequentes a dispneia, a restrição ventilatória mecânica e as alterações graves das trocas gasosas. Outros padrões de respostas podem ser encontrados na broncoconstrição induzida pelo exercício, na doença vascular pulmonar, na insuficiência cardíaca e em cardiopatias congénitas.

A avaliação cardiorrespiratória no exercício fornece ainda indicadores prognósticos em várias doenças. Descrevem-se neste documento vários trabalhos que estudaram os parâmetros indicadores de prognóstico em doenças como a DPOC, a doença intersticial pulmonar, a hipertensão pulmonar primária, a fibrose quística e a insuficiência cardíaca.

Este documento demonstra ainda a utilidade dos testes de exercício na definição das respostas às intervenções terapêuticas, em avaliações seriadas.

O grupo de trabalho envolvido neste documento considerou importante apresentar as indicações baseadas na evidência para a realização dos testes de exercício na prática clínica. A evidência actual é clara quanto à utilidade da prova de exercício cardiopulmonar, das provas de marcha e das provas de carga constante na avaliação do grau de intolerância ao exercício, do prognóstico e dos efeitos das intervenções terapêuticas em doentes adultos com doença pulmonar crónica (DPOC, doença intersticial pulmonar, hipertensão pulmonar primária), em crianças e adultos com fibrose quística, em crianças e adultos 
com broncospasmo induzido pelo exercício, em adultos com insuficiência cardíaca congestiva e em crianças e adolescentes com cardiopatias congénitas.

$\mathrm{Na}$ elaboração deste documento, os autores pretenderam fornecer as respostas às perguntas que se colocam com frequência na prática clínica:

- Quando se deve pedir uma avaliação da intolerância ao esforço?
- Qual o teste mais adequado?

- Quais as variáveis a seleccionar na avaliação do prognóstico de determinada doença ou na avaliação do efeito de uma intervenção terapêutica particular?

O documento contém ainda um suplemento que pode ser obtido on-line e que descreve as bases fisiológicas subjacentes aos parâmetros avaliados nas provas de exercício cardiopulmonar.

\section{$O$ índice de BODE formulado por Celli e colaboradores demonstrou ser um melhor índice preditivo de mortalidade em doentes com DPOC do que o FEV isoladamente}

\section{Comentário}

Este documento irá constituir uma referência importante na abordagem clínica das doenças respiratórias crónicas.

Para além da importância das provas de exercício cardiopulmonar na identificação das causas de intolerância ao esforço, aspectos já documentados em trabalhos anteriores ${ }^{1-6}$, este documento fornece-nos recomendações sobre o uso dos testes de esforço (CPET, prova de marcha e prova de shuttle) no estabelecimento de índices de prognóstico, na monitorização da evolução das doenças e na avaliação da resposta às intervenções terapêuticas. Segundo a literatura actual, variáveis como a capacidade para o exercício, avaliada em termos do consumo máximo de oxigénio $\left(V^{\prime} \mathrm{O}_{2 \text { pico }}\right)$ ou da distância percorrida na prova de marcha de seis minutos ${ }^{7-8}$, ou outras variáveis, como o limiar anaeróbico ou a saturação de oxigénio, consideram-se melhores factores preditivos do prognóstico do que os parâmetros da função respiratória ou da função cardíaca avaliados em repouso.

Estes testes estão indicados na avaliação prognóstica de doentes com DPOC, doença intersticial pulmonar, hipertensão pulmo- nar primária, fibrose quística, insuficiência cardíaca congestiva, em candidatos a transplante e a intervenções cirúrgicas torácicas. Eis alguns exemplos:

Nos doentes com DPOC, o consumo de oxigénio de pico $\left(V^{\prime} \mathrm{O}_{2 \text { pico }}\right)$ é considerado o factor preditivo de mortalidade aos 5 anos mais significativo. Hiraga e colaboradores reportaram uma mortalidade aos 5 anos de $62 \%$ em doentes com $\mathrm{VO}_{\text {2pico }}$ inferior a 10 $\mathrm{ml} / \mathrm{kg} / \mathrm{min}^{9}$.

O índice de BODE formulado por Celli e colaboradores ${ }^{10}$ demonstrou ser um melhor índice preditivo de mortalidade em doentes com DPOC do que o FEV isoladamente. $\mathrm{Na}$ avaliação do risco cirúrgico, em particular na cirurgia de ressecção pulmonar, o valor de consumo de oxigénio de $15 \mathrm{ml} / \mathrm{kg} / \mathrm{min}$ é indicado como o limiar abaixo do qual existe risco significativo de complicações perioperatórias ${ }^{11}$.

$\mathrm{Na}$ avaliação pré-operatória realizada por Geddes e colaboradores em doentes com enfisema candidatos a cirurgia de redução de volume pulmonar, uma distância inferior a 150 metros na prova de marcha constituiu um índice preditivo de elevada mortalidade perioperatória ${ }^{12}$. 
O National Emphysema Treatment Trial ${ }^{13}$ reportou que doentes com enfisema predominante nos lobos superiores candidatos a cirurgia de redução de volume pulmonar, com grande limitação no teste de exercício pré-operatório (inferior a 25 Watts nas mulheres e inferior a 40 Watts nos homens), apresentavam melhorias significativas na sobrevida e na avaliação funcional 3 anos após a cirurgia, quando se comparava com os resultados obtidos com o tratamento médico.

A prova de esforço cardiopulmonar e a distância percorrida na prova de marcha têm sido utilizadas em estudos clínicos que avaliam a resposta às intervenções terapêuticas em doentes com patologia pulmonar e cardíaca (exemplos: DPOC, doença intersticial pulmonar, fibrose quística, hipertensão pulmonar primária e insuficiência cardíaca congestiva). As provas de esforço têm sido amplamente utilizadas em patologia cardiorrespiratória para avaliar os efeitos a curto e a longo prazo da reabilitação com treino de exercício, da terapia hormonal e nutricional, da terapêutica farmacológica, da administração de oxigénio e de heliox e das intervenções cirúrgicas, como a cirurgia de redução de volume pulmonar e o transplante.

Os protocolos das provas de esforço com carga constante de alta intensidade (exemplo: 75 a $80 \%$ da potência máxima) são os mais indicados para demonstrar os efeitos positivos da terapêutica broncodilatadora, da administração de oxigénio e de heliox durante o exercício, da redução de volume pulmonar por broncoscopia e da reabilitação. Com estes protocolos são demonstrados, entre outros efeitos, um aumento significativo do tempo de endurance, a redução da dispneia e da hiperinsuflação pulmonar para a mesma carga, em exames sequenciais.
Quando não existe acesso às provas de esforço cardiopulmonares realizadas em laboratório, a avaliação do efeito da reabilitação, das intervenções farmacológicas, da oxigenoterapia e das intervenções cirúrgicas pode ser feita pelos testes de terreno, como a prova de marcha e a prova de shuttle. Redelmeier e colaboradores apontaram a distância de 54 metros na prova de marcha como a diferença clinicamente significativa no desempenho em doentes com DPOC após um programa de reabilitação ${ }^{14}$.

\section{Mensagem}

A prova de exercício cardiorrespiratória (CPET) é considerada o gold standard na avaliação das causas de intolerância ao exercício em doentes com doença cardíaca e pulmonar.

Estes testes estão indicados na avaliação prognóstica de doentes com DPOC, doença intersticial pulmonar, hipertensão pulmonar primária, fibrose quística, insuficiência cardíaca congestiva, em candidatos a transplante e a intervenções cirúrgicas torácicas.

A prova de esforço cardiopulmonar e as provas de marcha são instrumentos que avaliam a resposta às intervenções terapêuticas em doentes com patologia pulmonar e cardíaca, documentando os efeitos a curto e a longo prazo da reabilitação com treino de exercício, da terapia hormonal e nutricional, da terapêutica farmacológica, da administração de oxigénio e de heliox e das intervenções cirúrgicas, como a cirurgia de redução de volume pulmonar e o transplante.

\section{A prova de esforço cardiopulmonar e a distância percorrida na prova de marcha têm sido utilizadas em estudos clínicos que avaliam a resposta às intervenções terapêuticas em doentes com patologia pulmonar e cardíaca}




\section{Bibliografia}

1. American Thoracic Society; American College of Chest Physicians, ATS/ACCP Statement on Cardiopulmonary Exercise Testing. Am J Respir Crit Care Med 2003; 167 : 211-77.

2. ERS Task Force on Standardization of Clinical Exercise Testing with reference to lung diseases: indications, standardization and interpretation strategies. Eur Respir J 1997; 10: 2662-89.

3. Roca J, Whipp BJ (Eds.). Clinical Exercise Testing. Eur Respir Mon 1997; 6.

4. Wasserman K, Hansen JE, Sue DY, Stringer W, Whipp BJ (Eds.). Principles of Exercise Testing and Interpretation, $4^{\text {th }}$ Ed. Philadelphia, Lea \& Febiger, 2004.

5. Weisman IM, Zeballos RJ, eds. Clinical Exercise Testing. In Progress in Respiratory Research (Editor: CT Bolliger), Vol 32, Karger, Basel, 2002.

6. Rodrigues F. Estudo dos factores limitativos do exercício físico em doentes com doença pulmonar obstrutiva crónica. Rev Port Pneumol 2004; 1: 9-61.

7. Enright PL, McBurnie MA, Bittner V, et al. Cardiovascular Health Study. The 6-min walk test: a quick measure of functional status in elderly adults. Chest 2003; 123: 387-98. 8. Troosters T, Gosselink R, Decramer M. Six minute walking distance in healthy elderly subjects. Eur Respir J 1999; 14: 270-4.
9. Hiraga T, Maekura R, Okuda Y, et al. Prognostic predictors for survival in patients with COPD using cardiopulmonary exercise testing. Clin Physiol Funct Imaging 2003; 23: 324-31.

10. Celli BR, Cote CG, Marin JM, et al. The body mass index, airflow obstruction, dyspnoea, and exercise capacity index in chronic obstructive pulmonary disease. $\mathrm{N}$ Engl J Med 2004; 350: 1005-12.

11. Smith TP, Kinasewitz GT, Tucker WY, Spillers WP, George RB. Exercise capacity as a predictor of postthoracotomy morbidity. Am Rev Respir Dis 1984; 129 : 730-4.

12. Geddes D, Davies M, Koyama H, et al. Effect of lung-volume-reduction surgery in patients with severe emphysema. N Engl J Med 2000; 343: 239-45.

13. National Emphysema Treatment Trial Research Group. Cost effectiveness of lung-volume-reductionsurgery for patients with severe emphysema. N Engl J Med 2003; 348: 2092-102.

14. Redelmeier DA, Bayoumi AM, Goldstein RS, Guyatt GH. Interpreting small differences in functional status: the Six Minute Walk test in chronic lung disease patients. Am J Respir Crit Care Med 1997; 155: 1278-82.

Fátima Rodrigues 11-02-2007 